Saudi Journal of Pathology and Microbiology

Abbreviated Key Title: Saudi J Pathol Microbiol

ISSN 2518-3362 (Print) |ISSN 2518-3370 (Online)

Scholars Middle East Publishers, Dubai, United Arab Emirates

Journal homepage: http://scholarsmepub.com/sjpm/

\title{
Perineural Invasion in Prostatic Carcinoma as Detected by S100 and its Implication in Prognosis
}

\author{
Sneha Aditi ${ }^{*}$, Dr. Ram Das Naik MD², Dr. Anuradha C.K Rao MD DNB \\ ${ }^{1}$ Postgraduate, Department of Pathology, Yenepoya Medical College, Mangalore, India \\ ${ }^{2}$ Professor and HOD, Yenepoya Medical College, Mangalore, India \\ ${ }^{3}$ Professor, Yenepoya Medical College, Mangalore, India
}

DOI: $10.36348 /$ SJPM.2019.v04i10.006

| Received: 19.10.2019 | Accepted: 27.10.2019 | Published: 30.10.2019

*Corresponding author: Sneha Aditi

\section{Abstract}

Background and objectives: Carcinoma prostate is becoming an increasingly significant international health problem. Perineural tumour growth is associated with adverse prognostic features and a poor outcome; hence is a necessary component in histopathological reporting of malignancy. For accurate detection of perineural cancer spread, immunohistochemistry (IHC) with S100 was performed along with haematoxylin and eosin (H\&E) stain. This study attempts to correlate the clinic-pathological prognostic factors with PNI, in a regional scenario. Methods: A retrospective study was conducted at a tertiary care hospital over a period of one year. Specimens were trurcut biopsy, TURP chips and prostatectomy specimen, sent in $10 \%$ formalin. Paraffin embedded blocks was stained with H\&E and representative sections with S100. Relationship of tumor with nerve was established and graded accordingly as: Category 0: nerves without immediate tumor-cell-contact. All nerves being fully surrounded by tumor (classical perineural invasion) were categorized group 3. Two further categories discriminated between nerves that were touched either by carcinoma cells below $50 \%$ of the circumference (category 1) or above $50 \%$ as category 2. Results: Out of 30 cases of prostatic adenocarcinoma, majority of specimens were needle biopsies (47\%). The age of presentation were 55-84years with mean age being 68.93 years. Pretreatment and posttreatment PSA levels were assessed. Most common presentation was bladder outlet obstruction $(57 \%)$ and treatment given was radical Prostatectomy (44\%). Perineural invasion (PNI) was seen in $66.7 \%$ of cases and correlated positively with Gleason's score, pT categories, \%PSA. Perineural spread noted in $73.3 \%$ cases and negatively correlated with Grade group.

Keywords: Adenocarcinoma Prostate, PSA, S100, Perineural Invasion, Perineural spread, Gleason's Score, Grade group. Copyright @ 2019: This is an open-access article distributed under the terms of the Creative Commons Attribution license which permits unrestricted use, distribution, and reproduction in any medium for non-commercial use (NonCommercial, or CC-BY-NC) provided the original author and sources are credited.

\section{INTRODUCTION}

Perineural invasion (PNI) defined as tumor cell invasion in, around and through the nerves, is a highly prominent feature in prostatic carcinoma[1] Studies have noted PNI in $75 \%$ of radical prostatectomy specimens and in up to $25 \%$ of needle biopsies from patients without lymph node metastasis[2].

The compartment situated between the nerve axons, supporting cells, tissues and the epineuria is the perineural space. Local microenvironment creates a complex interaction when tumor cells invade perineural space [3]. This space provides a channel for tumor spread beyond the original site of tumor occurrence. Perineural tumor growth is of two types: when it is affecting small unnamed nerves it signifies PNI; and when it is affecting larger nerves it shows perineural spread (PNS) and presents with clinical symptoms related to the involved nerve. Both forms of perineural tumor growth presents with adverse prognostic features and are necessary component of the histopathological reporting of malignancies [3]. The accepted mechanism for spread of cancer cells within the perineural space is PNI suggesting an indication for extra prostatic spread [4].

PNI in prostatic carcinoma is associated with poor outcome; decreased overall survival and an increased recurrence rate. Saeter et al. showed biopsy cores showing PNI positivity $>50 \%$ was associated with higher prostate cancer induced mortality, independent of other clinic- pathological parameters [5].

For the accurate detection of perineural cancer spread, S100 is performed in addition to the standard haematoxylin and eosin $(\mathrm{H} \& \mathrm{E})$ staining techniques for specimens. 


\section{OBJECTIVES}

To demonstrate the role and significance of $\mathrm{S} 100$ as a marker in assessing the PNI in prostatic carcinoma and correlation of PNI with clinicpathological parameters including prostatic specific antigen (PSA) levels, radiological evidence of metastasis and histopathological parameters.

\section{MATERIALS AND METHODS}

A retrospective study was conducted at a tertiary care hospital in which 30 prostate carcinoma cases received over one year period were included after obtaining ethical clearance from the university. The patient data, PSA levels when available, postoperative histopathological and therapy reports were collected and reviewed. For 15 patients follow up data was available. In $13(43.3 \%)$ patients bilateral orchidectomy was performed.

The median age of the patients at the time of diagnosis was 68.93 years (range 55-84 years). During follow up 2 patients expired. Thirty specimens were fixed in ten percent formalin, stained with Hematoxylin and Eosin ( $\mathrm{H}$ and $\mathrm{E}$ ) for histopathological study. A further section from tumor was taken and immunostaining was performed using S100 (polyclonal antibody 1; 1:2000; Pathinsitu). Slides were reviewed with an Olympus CH20i microscope.

Examination of various categories and its relationships with tumor and nerves are done. Category 0 : normal nerves surrounded by tumour cells but without immediate contact of tumour epithelia with the perineurium. Category 1: tumour epithelia touching the nerve, but below $50 \%$ of the circumference. Category 2: nerve-tumour contact above 50\%. Category 3: Classical Perineural invasion (PNI), in which the nerves are circumferentially surrounded by carcinoma cells.

Data was entered into Microsoft excel data sheet and analyzed using SPSS 23 version software. All results were considered significant at $\mathrm{p}$ values $<0.05$.

\section{RESULTS}

Amongst the 30 cases evaluated the mean patient age was 68.93 years (range 55-84 years). PSA levels were not recorded for 2 patients. The mean total prostatic specific antigen (tPSA ) level before treatment was $100.08 \mathrm{ng} / \mathrm{mL}$ (range 4.87 to $1564 \mathrm{ng} / \mathrm{mL}$ ). The mean free prostatic specific antigen (fPSA) and percentage prostatic specific antigen (\%PSA) before treatment was $20.27 \mathrm{ng} / \mathrm{mL}$ and $24.31 \%$ and standard deviation was 37.28 and $20.44 \%$ respectively.

Post treatment PSA levels were recorded for only 15 patients. The mean tPSA, fPSA and $\%$ PSA post treatment were $22.02 \mathrm{ng} / \mathrm{mL}, 7.44 \mathrm{ng} / \mathrm{mL}$ and
$28.44 \%$. The different specimens received were trurcut biopsy (47\%), transurethral resection of prostate chips (TURP) (26\%) and prostatectomy specimen (27\%).

Patients most commonly presented with bladder outlet obstruction (BOO) with 57\%, followed by $36 \%$ prior prostatic biopsy proven carcinoma cases. Two $(6 \%)$ cases presented with history of bladder carcinoma.

The most common treatment provided were radical prostatectomy in $46 \%$ cases, Channel TURP in $40 \%$ and chemotherapy in $13 \%$ cases. For $47 \%$ cases bilateral orchidectomy was performed for androgendeprivation therapy as additional workup.

Histopathological examination of $\mathrm{H}$ and $\mathrm{E}$ stained slides was initially carried out. Nerves were noted in $76.7 \%$ cases where as with the use of S100 IHC stain nerve were seen in $96.7 \%$ cases. PNS in $\mathrm{H}$ and $\mathrm{E}$ stained slides was noted in $46.7 \%$ whereas 73.3 $\%$ cases showed PNS when stained by S100.

Similarly PNI was only seen in $26.7 \%$ cases by Hand E stain where as by $\mathrm{S} 100$ stain $66.7 \%$ cases showed invasion. When PNI was assessed Category 0 was seen in $43 \%$ cases. Category1 in $20 \%$ cases, category 2 in $17 \%$ cases and category 3 in $20 \%$ cases.

All radical prostatectomy cases showed PNI. Higher stage (pT3Nx) cases showed more of category 3 PNI (80\%). Lower tumour stages however had all categories of PNI.

PNS was seen in $73 \%$ cases. PNI with PNS was noted in $50 \%$ cases. Category 1 PNI showed more number of cases with PNS followed by classical PNI along with category 3 .

Table-1: Perineural spread vs PNI Category

\begin{tabular}{|c|c|c|c|c|c|}
\hline \multicolumn{6}{|l|}{ Count } \\
\hline \multirow[t]{2}{*}{ Perineural spread } & \multicolumn{4}{|c|}{ PNI category } & \multirow[t]{2}{*}{ Total } \\
\hline & .0 & 1.0 & 2.0 & 3.0 & \\
\hline present & 7 & 6 & 4 & 5 & 22 \\
\hline Absent & 6 & 0 & 1 & 1 & 8 \\
\hline Total & 13 & 6 & 5 & 6 & 30 \\
\hline
\end{tabular}

PNI: Perineural invasion

The most common Gleason's score is 9 is seen in $47 \%$ cases followed by Gleason's score 7 seen in $27 \%$ cases. A negative correlation (p value -0.049 ) was seen between PNI category and grade group stating that as grade group increase the category decreases. Hence, lower grade cases have more PNI. But there was no correlation between category and Gleason's score. 


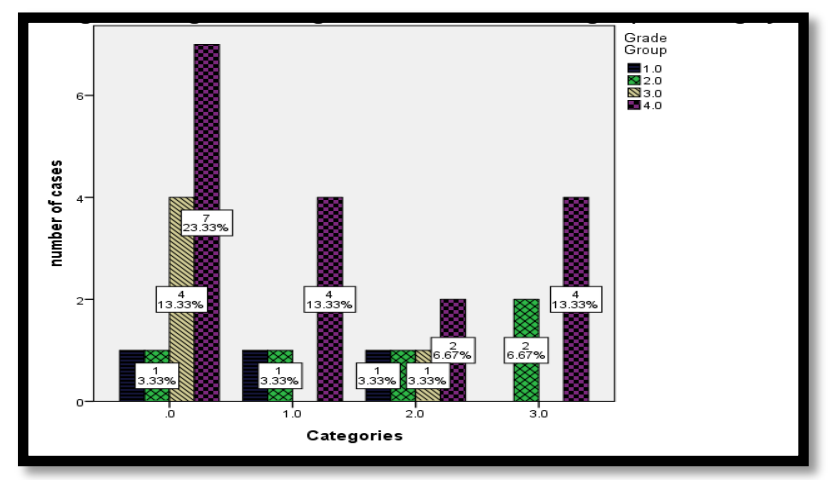

Fig-1: Bar diagram showing correlation between grade group and category

A strong positive correlation was observed between PNI and Gleason's Score (P value: 0.05). Whereas no correlation was seen with Grade group.

Similarly a negative correlation was seen between PNS and Grade group (P value -0.043). No correlation was noted with Gleason's score. This effectively meant that a higher grade group was associated with lesser PNS.

Percentage PSA value $>25 \%$ was considered as normal where as $<25 \%$ was considered as a possibility of malignancy. $60 \%$ cases showed percentage PSA $<25 \%$ and $40 \%$ cases showed $>25 \%$ percentage PSA.

There is weak correlation with categories and \%PSA (P value: 0.8). However there was no significant correlation between tPSA level and fPSA levels.

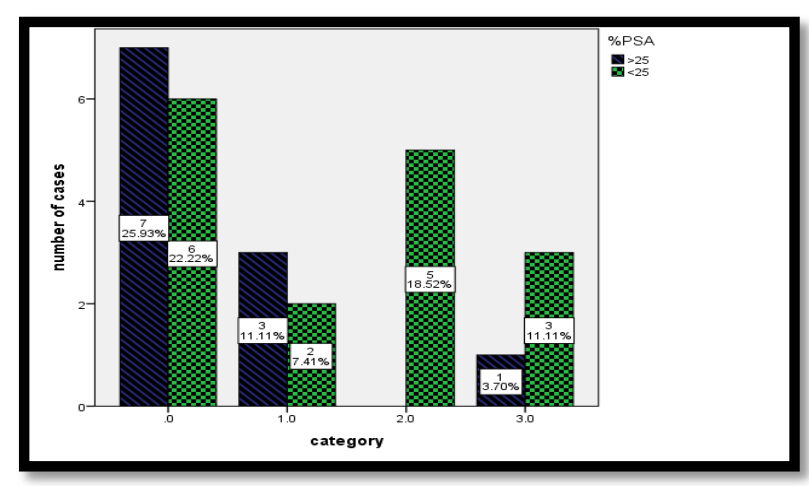

Fig-2: Bar diagram between \% PSA and PNI category

\%PSA: Percentage Prostate specific Antigen, PNI: Perineural invasion. A strong correlation was seen between PNI and \% PSA (p value: 0.012) level however no correlation was seen with PNS. PNI and PNS had no correlation with other PSA parameters like tPSA and fPSA.
Table-2: \% PSA correlation with PNI, PNS and Metastasis

\begin{tabular}{|c|c|c|c|}
\hline & \multicolumn{3}{|c|}{ \%PSA } \\
\hline & $>25(n=11)$ & $<25(n=16)$ & $P$ value \\
\hline PNI & & & \\
\hline Present $(n=20)$ & 7 & 10 & 0.012 \\
\hline Absent $(n=8)$ & 4 & 6 & \\
\hline PNS & & & \\
\hline Present $(n=22)$ & 9 & 10 & 0.203 \\
\hline $\operatorname{Absent}(\mathrm{n}=8)$ & 2 & 6 & \\
\hline Metastasis & & & \\
\hline Present (n=7) & 2 & 4 & \\
\hline Absent $(n=23)$ & 9 & 12 & 0.081 \\
\hline
\end{tabular}

PNI: Perineural invasion, PNS: Perineural spread, $\%$ PSA: Percentage Prostatic specific Antigen

Metastasis was noted in $23 \%$ cases. Metastasis shows a weak correlation with \%PSA level (0.08). However there was no correlation seen between category, Gleason's score, Grade group, tPSA and fPSA levels.

There was a highly significant decrease in tPSA and fPSA levels after treatment. Paired Samples Test was done to assess the difference in tPSA, fPSA and \% PSA levels before vs after treatment. Serum tPSA before treatment and tPSA after treatment is highly significant.

A significant strong correlation was seen between \% PSA level postoperatively with PNI ( $p$ value: 0.018 ), Gleason's score ( $p$ value: 0.018 ), Grade group ( $\mathrm{p}$ value: 0.035 ). However no correlation was noted with PNI category and metastasis.

A weak correlation was seen between levels of fPSA post operatively with Grade group ( $\mathrm{p}$ value: 0.077). However no correlation was seen with other parameters. Similarly no correlation was noted with tPSA level.

No follow up was seen in $50 \%$ cases after treatment. $30 \%$ patients were normal with no abnormality found ultrasonographically. $2(7 \%)$ patients expired and had no PNI and no bony metastasis but showed high tumor stage PT3. Other complains were urinary incontinence, urinary bladder thickness with hydronephrosis and vesicoureteric junction obstruction secondary to extension of tumor was noted in rest $13 \%$ patients. 


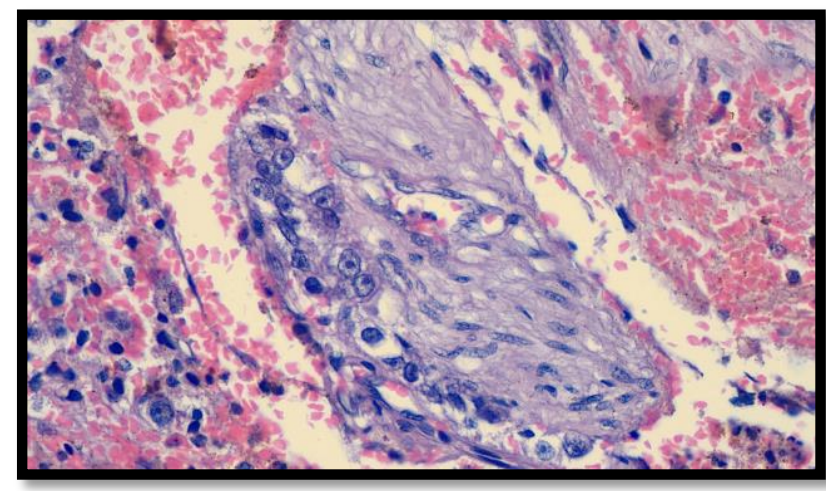

Fig-3: Category 1 tumor cells touching the nerve, but $<$ $50 \%$ of the circumference.

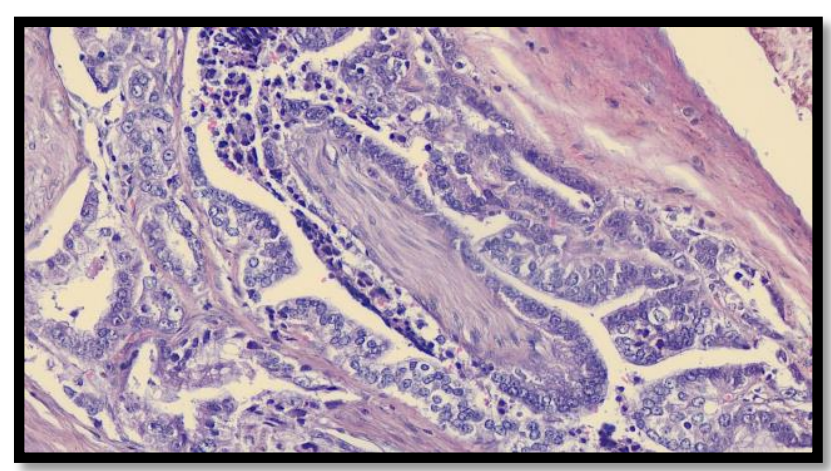

Fig-4: Category 2: nerve-tumor cells are in contact $>\mathbf{5 0} \%$ (40x)

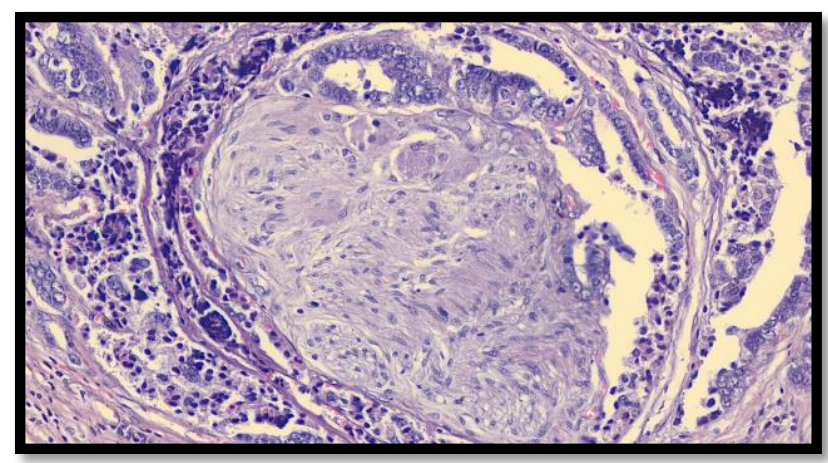

Fig-5: Category 3: distinctive examples of classical perineural invasion where the nerves are circumferentially surrounded by carcinoma cells $(40 x, H \& E)$

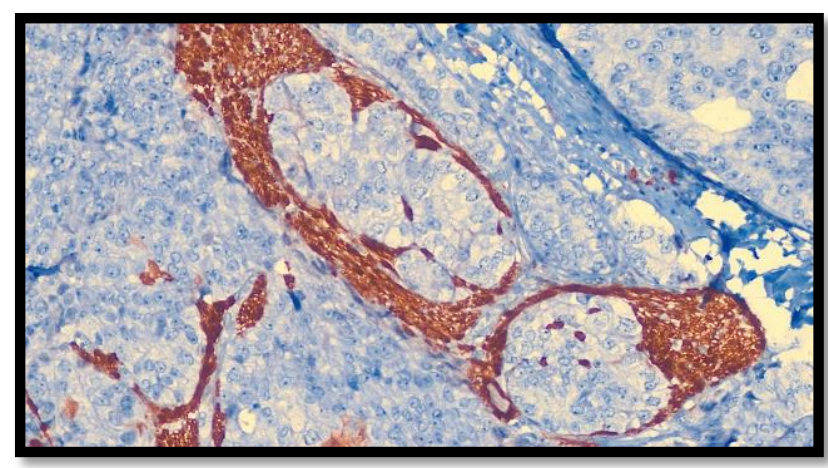

Fig-6: Category 3: Perineural invasion in S100 (40X)

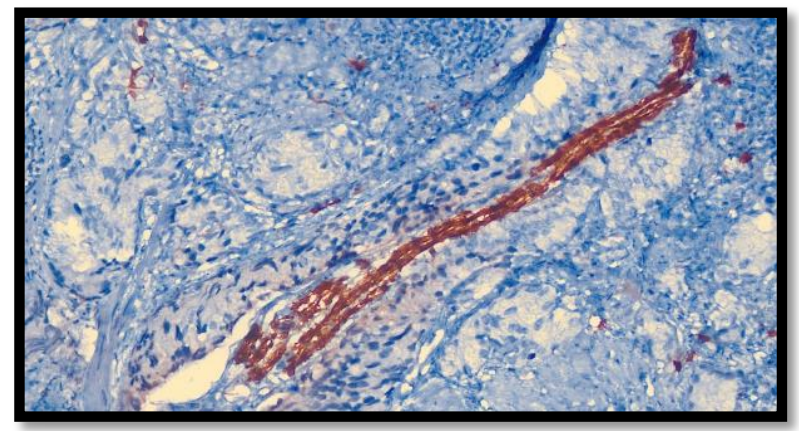

Fig-7: Category 2: tumour contact above $50 \%$ in S100.(40x)

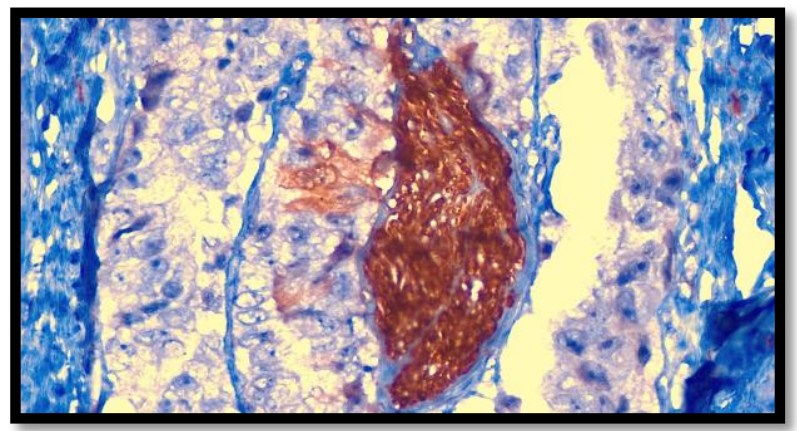

Fig-8: Category 1: tumour contact below $50 \%$ in S100.(40x)

\section{DISCUSSION}

Prostate cancer is a nerve inclined malignancy, which advances by PNI. Prognostic parameters of prostate cancer in different specimen such as prostatectomy specimen, biopsy specimen and TURP are age, Gleason's score, Grade group, PSA levels and pathological stage in prostatectomy specimen. PNI positivity as an important prognostic factor requires further investigation to evaluate it as prognostic factor. Other histological factors are being studied to identify predictors of prognosis.

The most common affected males in our study belonged to the age group of 55-84yrs. The normal level of tPSA level is $<4 \mathrm{ng} / \mathrm{mL}$, FPSA level is $<0.01 \mathrm{ng} / \mathrm{mL}$ whereas percentage PSA is $>25 \%$ [8]. In case of carcinoma there is up to 10 folds increase in tPSA level[8]. In our study the mean tPSA level was $100.08 \mathrm{ng} / \mathrm{mL}$ (range 4.87 to $1564 \mathrm{ng} / \mathrm{mL}$ ) with standard deviation of 287.89 . The mean fPSA and $\%$ PSA are $23.27 \mathrm{mg} / \mathrm{dl}$ and $24.31 \%$. The treatment effect on PSA in our study is highly significant as the mean pretreatment tPSA was $100.08 \mathrm{ng} / \mathrm{mL}$ which reduced to $22.02 \mathrm{ng} / \mathrm{mL}$ after treatment. However posttreatment tPSA levels were recorded in $50 \%$ patients only.

In our study patients presented most commonly with BOO (46\% cases) similar to Hamilton W et al. who stated that four symptoms such as urinary retention, frequency, hesitancy and nocturia, probably represent enlargement of the prostate gland. Retention 
had the strongest association of these with prostatic cancer [10].

The treatments provided in this study were radical prostatectomy (44\%), Channel TURP (43\%) and chemotherapy (13\%). For $47 \%$ cases bilateral orchidectomy was performed along with other treatment modalities as androgen-deprivation therapy. Surgical and medical castration is considered to increase overall survival rate [11]. Rud O et al. stated that advantages of orchidectomy are rapidly effective in lowering testosterone level which is achieved between 3 to 12 hours after surgery and is related with good compliance [12]. Goldenberg et al. added that this form of therapy improves quality of life when the patient is androgen hormone receptor sensitive by giving them life with therapy free interval $[13,14]$.

The significance of $\mathrm{S} 100$ staining for appreciation of nerves can be seen by the fact that the nerves which were noticeable in only $70 \%$ in Hand $\mathrm{E}$ stained sections were noticed in $96.7 \%$ cases on $\mathrm{S} 100$ staining in this study. Similarly PNS and PNI were also noted more aptly with S100 staining. The staining of nerve by $\mathrm{S} 100$ was appropriately categorized into 0,1 , 2 and 3 to evaluate the quality and quantity of perineural affection by tumor cells. The cancer cells that touch nerve tissue may just start infiltrating into nerve sheaths and was categorized a positive tumournerve interaction by the degree of circumferential involvement ( $1=$ surrounding $<50 \% ; 2=$ surrounding $>50 \%$ but not the complete circumference; $3=$ classical perineural invasion, $100 \%$ circumference)[2].

Batsakis et al. also offered a broad definition of PNI, characterizing it as tumor cell invasion in, around, and through the nerve tumor in close proximity to nerve and involving at least $33 \%$ of its circumference or tumor cells within any of the 3 layers of the nerve sheath [6]. When PNI was assessed categorically in our study it was noted that Category 0 was seen in $43 \%$ cases, Category1 in $20 \%$ cases, category 2 in $17 \%$ cases and category 3 in $20 \%$ cases. A similar study by Lubig S. et al. showed $81.59 \%$ (category 0), 6.89\% (category 1), $2.88 \%$ (category 2 ) and $1.79 \%$ (category 3)[2].

All radical prostatectomy (44\%) cases showed PNI. PNI was noted in $66.7 \%$ of total cases in our study which was almost similar to Lubig S.et al. study where PNI is found in $53.5 \%$ of cases.

Higher stage (pT3Nx) cases showed more of category 3 Perineural invasion (80\%).However PNI was seen all tumour stages. Merrilees et al. in their study showed PNI in $90 \%$ cases with tumor stage T1 (71\%), T2 (28\%) T3 (1\%) [7], Jeon et al. in their study stated that PNI was seen in $42 \%$ cases with tumor stage T1 (15.8\%), T2 (20.5\%), T3 (25\%) and Lee et al. stated
PNI in $52.1 \%$ cases with tumor stage T2(70\%) T3 $(30 \%)$ [9].

A negative correlation ( $p$ value -0.049) was seen between PNI category and grade group, whereas a strong Positive correlation was observed between PNI and Gleason's Score (P value: 0.05 ) which was similar in study performed by Khoddami M. et al. where positive relation was seen between tumor score and Perineural invasion $(\mathrm{P}-$ value $=0.032)[17]$.

Metastasis was noted in 7 cases out of 30 cases in our study. There was negative correlation seen between metastasis and PNI in our study and no correlation was noted with tPSA, fPSA; however weak association was noted with \%PSA (p value 0.08 ). No association was present between Gleason's score and Gleason's grade in our patients. Hirobe et al. examined 366 patients with 28 showing metastasis. They found that PSA, Gleason's score, clinical stage, and percentage of positive biopsy cores were all associated with bone metastasis [16].

Follow up of patient after 6 months was recorded with only $50 \%$ patients being followed up with $30 \%$ patients performing well and $7 \%$ patients expired. However in our study category 0 was seen in the patient that expired stating that PNI and metastasis was not certain cause for patient mortality, whereas Saeter et al. in their study stated that biopsy cores with $>50 \%$ PNI positivity can be associated with increased risk for prostate cancer mortality which is independent of other clinic-pathological parameters[5].

\section{CONCLUSION}

This study highlights the importance of S100 in assessing PNI in prostatic carcinoma. Higher Gleason's scores were associated with a higher category of PNI and lower \%PSA. However the same has to be juxtopolated on a study of larger dimension to validate the use of PNI as a prognostic indicator in prostatic carcinomas.

\section{REFERANCES}

1. Batsakis, J. G. (1985). Nerves and neurotropic carcinomas. The Annals of otology, rhinology, and laryngology, 94(4 Pt 1), 426-427.

2. Lubig, S., Thiesler, T., Müller, S., Vorreuther, R., Leipner, N., \& Kristiansen, G. (2018). Quantitative perineural invasion is a prognostic marker in prostate cancer. Pathology, 50(3), 298-304.

3. Liebig, C., Ayala, G., Wilks, J. A., Berger, D. H., \& Albo, D. (2009). Perineural invasion in cancer: a review of the literature. Cancer: Interdisciplinary International Journal of the American Cancer Society, 115(15), 3379-3391.

4. Brown, I. S. (2016). Pathology of perineural spread. Journal of Neurological Surgery Part B. Skull Base, 77(02), 124-130. 
5. Sæter, T., Bogaard, M., Vlatkovic, L., Waaler, G., Servoll, E., Nesland, J. M., ... \& Axcrona, U. (2016). The relationship between perineural invasion, tumor grade, reactive stroma and prostate cancer-specific mortality: A clinicopathologic study on a population-based cohort. The Prostate, 76(2), 207-214.

6. Batsakis, J. G. (1985). Nerves and neurotropic carcinomas. The Annals of otology, rhinology, and laryngology, 94(4 Pt 1), 426-427.

7. Masieri, L., Lanciotti, M., Nesi, G., Lanzi, F., Tosi, N., Minervini, A., ... \& Serni, S. (2010). Prognostic role of perineural invasion in 239 consecutive patients with pathologically organ-confined prostate cancer. Urologia internationalis, 85(4), 396-400.

8. Atan, A., \& Güzel, Ö. (2013). How should prostate specific antigen be interpreted?. Turkish journal of urology, 39(3), 188.

9. Jeon, H. G., Bae, J., Yi, J. S., Hwang, I. S., Lee, S. E., \& Lee, E. (2009). Perineural invasion is a prognostic factor for biochemical failure after radical prostatectomy. International journal of urology, 16(8), 682-686.

10. Hamilton, W., Round, A., Sharp, D., \& Peters, T. J. (2005). Clinical features of colorectal cancer before diagnosis: a population-based case-control study. British journal of cancer, 93(4), 399.

11. Maatman, T. J., Gupta, M. K., \& Montie, J. E. (1985). Effectiveness of castration versus intravenous estrogen therapy in producing rapid endocrine control of metastatic cancer of the prostate. The Journal of urology, 133(4), 620-621.
12. Rud, O., Peter, J., Kheyri, R., Gilfrich, C., Ahmed, A. M., Boeckmann, W., ... \& May, M. (2012). Subcapsular orchiectomy in the primary therapy of patients with bone metastasis in advanced prostate cancer: An anachronistic intervention?. Advances in urology, 2012.

13. Goldenberg, S. L., Bruchovsky, N., Gleave, M. E., Sullivan, L. D., \& Akakura, K. (1995). Intermittent androgen suppression in the treatment of prostate cancer: a preliminary report. Urology, 45(5), 839845.

14. Bruchovsky, N., Rennie, P. S., Coldman, A. J., Goldenberg, S. L., To, M., \& Lawson, D. (1990). Effects of androgen withdrawal on the stem cell composition of the Shionogi carcinoma. Cancer research, 50(8), 2275-2282.

15. Merrilees, A. D., Bethwaite, P. B., Russell, G. L., Robinson, R. G., \& Delahunt, B. (2008). Parameters of perineural invasion in radical prostatectomy specimens lack prognostic significance. Modern Pathology, 21(9), 1095.

16. Hirobe, M., Takahashi, A., Hisasue, S. I., Kitamura, H., Kunishima, Y., Masumori, N., \& Tsukamoto, T. (2007). Bone scanning-who needs it among patients with newly diagnosed prostate cancer?.Japanese journal of clinical oncology, 37(10), 788-792.

17. Khoddami, M., Khademi, Y., Aghdam, M. K., \& Soltanghoraee, H. (2016). Correlation between Gleason scores in needle biopsy and corresponding radical prostatectomy specimens: a twelve-year review. Iranian journal of pathology, 11(2), 120. 